\section{CALL FOR INCREASED PUBLIC INVOLVEMENT IN THE NHS}

The Department of Health is issuing a call for healthcare professionals to support Local Involvement Networks (LINks).

LINks are made up of individuals and community groups who work together to improve local services and cover all health and social care services that are commissioned by the NHS and local authorities in an area. The only services that are excluded are children's social services.

With the launch of the 150th LINk in early 2009, the Department of Health is advocating that healthcare staff from all sectors proactively engage with their LINk and encourage twoway conversations between services and patients.

Joan Saddler, National Director of Patient and Public Affairs at the Department of Health, said 'From the beginning of next year, all organisations providing NHS care in England will be required, by law, to take account of the NHS Constitution when making decisions and taking actions. It is vitally important that we not only raise awareness of the Constitution, but also make the public aware of the different ways in which they can become involved. As such, we are urging health and care professionals from GPs to nurses, dentists to community support workers and social workers, to proactively support their LINk by promoting participation amongst a range of diverse service users and by using their LINk as a vital service planning and delivery tool.

The call from the Department of Health comes as part of a nationwide campaign that aims to drive up awareness of LINks and promote their ability to help bring about genuine change. As part of this campaign, a LINks website has been launched on NHS Choices (www.nhs.uk/links) to alert the public, commissioners and service providers to the different ways they can get involved.

\section{DENTAL RESEARCH AWARD WIN}

A $£ 1.7 \mathrm{M}$ trial funded by the Health Technology Assessment (HTA) theme of the National Institute of Health Research will see if a prevention package delivered by dentists in practice can prevent tooth decay in young children. The outcomes could inform the development of NHS dental services and interventions in the UK.

The three-year multi-collaborative trial will take place in Northern Ireland but will involve a team of dental experts that will be led by Professor Martin Tickle of the Oral Health Unit at The University of Manchester.

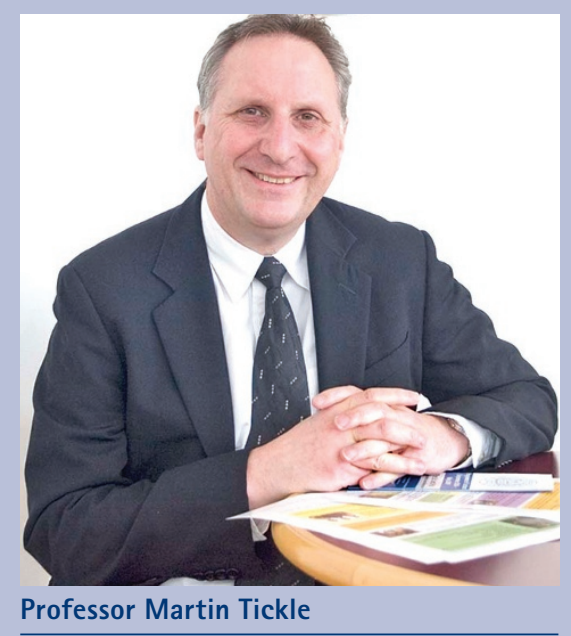

Recent studies have shown that prevention of decay in the primary teeth in NHS general dental practice is not very effective and that over a three year period 35\% of two- to three-year-olds registered with a dentist develop tooth decay. Northern Ireland has a particular problem as approximately $45 \%$ of five-year-olds have tooth decay.

In England all NHS general dental practitioners have been sent an evidence-based toolkit which identifies best evidence for preventive care, but research has yet to show whether these interventions are cost effective when used in every day NHS practice. The trial will therefore test the cost effectiveness of fluoride varnish and family-strength fluoride toothpaste provided in general practice twice a year to help prevent decay.

Professor Tickle said, 'The aim of the trial is to see if we can keep a larger proportion of children free of decay by using a fluoride varnish and toothpaste. Hopefully the findings will help to inform future policy on children's dental health and focus on proactively preventing tooth decay rather than treating the disease once it has started.'

\title{
SUCCESSFUL COLLABORATION SCOOPS PRIZE
}

A research collaboration between Professor Ian Needleman at the UCL Eastman Dental Institute, Professor Helen Worthington at the University of Manchester, the Cochrane Oral Health Group and the Universities of Bonn and Schleswig-Holstein has been awarded 2nd place in the 2009 DGP Meridol Award.

The research 'Full-mouth disinfection for the treatment of chronic periodontitis', published in the Journal of Clinical Periodontology (2008; 35: 591-604) investigated non-surgical approaches to managing periodontal diseases. A key finding was that new approaches to treatment, such as one visit full mouth debridement, were as effective as traditional methods of care.

The 2009 DGP Meridol Award represents another achievement for the Cochrane Oral Health Group, part of a worldwide non-profit organisation providing up-to-date information about the effects of oral health care.

The prize is also another achievement for the International Centre for Evidence-Based Oral Health (ICE$\mathrm{BOH})$, which is based in the Unit of Periodontology at the Eastman. The research had previously been awarded the German Cochrane Centre Prize for 2007. 